\title{
Carbon Dots with Tunable Photoluminescence Properties
}

\author{
Longyi Chen, Jin Zhang \\ Department of Chemical and Biochemical Engineering \\ University of Western Ontario \\ London, Ontario, Canada, N6A 5B9 \\ lchen449@uwo.ca; jzhang@eng.uwo.ca
}

\begin{abstract}
A facile process for producing carbon dots with tunable luminescence properties has been developed by using microwaveassisted technique. Citric acid and L-histidine were used as carbohydrate precursors. By varying the precursors concentration in ethylene glycol, adjustable multicolor carbon dots of blue, green to yellow under UV excitation were easily obtained. We found that the photoluminescence of carbon dots (diameter $<40 \mathrm{~nm}$ ) can be adjusted by simply varying precursor amount, which has not yet been reported before. Other methods for preparing different photoluminescent carbon dots often use oxidative/reductive chemicals, our method is facile and environmental friendly. The tuneable photoluminescence range can extend from $430 \mathrm{~nm}$ to $550 \mathrm{~nm}$ in this system.
\end{abstract}

Keywords: Carbon Dots, Microwave-Assisted, Tuneable Photoluminescence Properties.

\section{Introduction}

Carbon dots are a new member of carbon nanomaterials. [1] Their attracted properties includes good biocompatibility, inertness, facile surface modification and special photoluminescent properties. [2, 3] Various synthetic approaches has been explored for preparing carbon dots from bottom-up to top-down paths. [4, 5] Due to carbon dots favorable fluorescence and low toxicity, they have been broadly applied in areas of biosensing and bioimaging etc. [6-10] The photoluminescence mechanism of carbon dots is still at open issue among investigators. Roughly three kinds of explanations have been proposed for carbon dots particular fluorescent behaviors, namely quantum size effect, surface state and molecule state/carbon core state. [11]

Microwave-assisted synthesis and modification of carbon nanomaterials has been explored due to carbon materials strong interaction with microwave radiation. [12] Microwave represent energy-saving and time-saving strategy for carbon dots synthesis. Other common methods for carbon dots like hydrothermal often requires several hours or days, high temperature and high pressure. Many research groups used microwave to fast synthesize carbon dots. [13-17] Synthesized carbon dots either showed excitation-dependent or excitation-independent fluorescence. For multicolour carbon dots preparation, often changing the reaction parameters like reaction temperature, reaction additives, $\mathrm{pH}$, reaction time etc. are applied or different precursors, doping elements are used. [18-21]

Herein, we develop a facile strategy of synthesising photoluminescent adjustable carbon dots from blue, green to yellow under $312 \mathrm{~nm}$ UV excitation by varying precursors' concentrations. Zhu et al[22] has prepared varied precursor concentrations carbon dots, but the fluorescence change of the carbon dots is very little within $10 \mathrm{~nm}$. Our method can tune carbon dots emission shift up to $80 \mathrm{~nm}$ (sample 1 at $430 \mathrm{~nm}$ and sample 4 at $511 \mathrm{~nm}$ ). Fluorescent nanomaterials are affected by various parameters like surface state,[23] $\mathrm{pH}$, temperature etc. in our carbon dots synthesis, the difference of all samples is the precursor concentration which can cause different carbon dots surface states. The main reason causing this phenomenon could be the different precursors to ethylene glycol ratio. Ethylene glycol is a reductive agents in the polyol synthesis[24] which acts both as reaction solution and reductive agents. Comparatively, keeping ethylene glycol same in all the microwave assisted synthesis, the higher precursor concentration environment are less reductive compared to lower precursor concentrations. As a result, the higher precursor carbon dots has a higher oxidative surface state, therefore they are red-shifted. This speculation is in consistent with other references.[21, 25] A higher oxidative surface state of carbon dots can cause the red-shift phenomenon.

At the same time, even though different colour carbon dots display different colours under $312 \mathrm{~nm}$ UV excitation, each of them also behaves the typical excitation-dependent full colour emissions. Although the mechanism for this 
photoluminescence change has not been fully explained. This facile synthesis of concentration-induced multicolour carbon dots provide a multicolour platform for many applications like display, printing inks, and biosensors.

\section{Experimental}

\subsection{Materials}

Citric acid (251275), L-histidine (H8000, $\geq 99 \%)$, 3-Aminophenylboronic acid monohydrate (287512, 98\%), dialysis tube (PRUG10020, MWCO $1 \mathrm{kDa}$ ) were purchased from Sigma-Aldrich. Ethylene glycol was purchased from Caledon laboratories Ltd. All chemicals were used as received. Water of $18.2 \Omega$ resistivity was used throughout experiment.

\subsection{Preparation of Carbon Dots}

A polyol microwave assisted method was adopted in the preparation of carbon dots.[26, 27] For synthesizing the carbon dots used for the glucose biosensor, a typical process is as following: in a $20 \mathrm{~mL}$ vial, $100 \mathrm{mmol}$ ethylene glycol, $0.5 \mathrm{mmol}$ L-histidine and $0.5 \mathrm{mmol}$ citric acid were added and mixed well. Then the vial was put in a home microwave oven and heated for 20 minutes. The deep brown color indicated the formation of carbon dots. The solutions was dialyzed against water and freeze-dried to obtain powder samples. The powder samples were stored in 4 degrees $\mathrm{C}$.

For synthesizing a series of photoluminescent changing carbon dots, the precursor amount is as shown in table 1 : sample 1 (100 mmol ethylene glycol, $0.1 \mathrm{mmol}$ L-histidine and $0.1 \mathrm{mmol}$ citric acid), sample 2 (100 mmol ethylene glycol, $0.3 \mathrm{mmol}$ L-histidine and $0.3 \mathrm{mmol}$ citric acid), sample 3 (100 mmol ethylene glycol, $0.5 \mathrm{mmol}$ L-histidine and $0.5 \mathrm{mmol}$ citric acid), sample 4 (100 mmol ethylene glycol, $1 \mathrm{mmol} \mathrm{L}$-histidine and $1 \mathrm{mmol}$ citric acid) and sample 5 (100 mmol ethylene glycol, $3 \mathrm{mmol}$ L-histidine and $3 \mathrm{mmol}$ citric acid).

\subsection{Characterizations}

The fluorescent emission spectrum of carbon dots aqueous solutions were measured by fluorophotometry (QuantaMsterTM 30, PTI). The size and morphology of the carbon dots were measured by transmission electron microscope (TEM, Philips CM-10 transmission electron microscope operating at $80 \mathrm{kV}$ ). UV-vis spectrum were obtained by Agilent Cary $60 \mathrm{UV}-\mathrm{Vis}$.

\section{Results and Discussion}

\subsection{Photoluminescent Tuneable Carbon Dots}

Varied optical carbon dots solution were prepared in the concentration of $0.1 \mathrm{mg} / \mathrm{mL}$. Figure 1 shows the carbon dots solution under ambient light and under the UV panel of $312 \mathrm{~nm}$ excitation light. In the ambient environment, slightly yellow colour change are observed and the colour deepens from sample 1 to sample 5. Under the UV panel excitation (312 $\mathrm{nm}$ ), changing colour emission were presented from blue emission to green emission to light yellow colour.

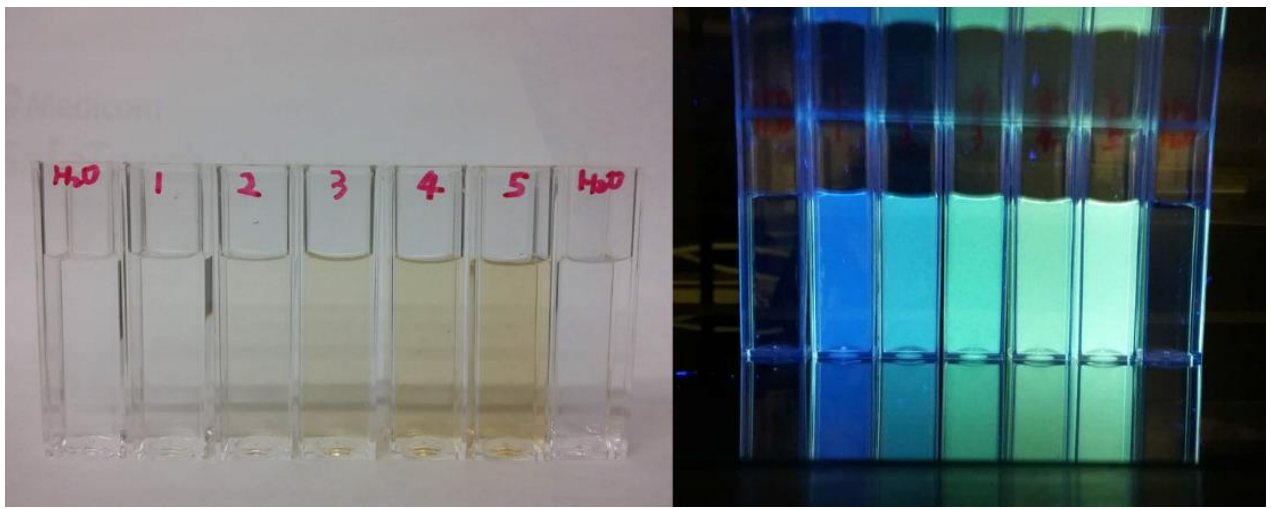

Fig. 1: Photo image of color tunable carbon dots under room light and excited by $312 \mathrm{~nm} \mathrm{UV}$ panel.

The fluorescence spectra of the carbon dots are recorded as shown in figure 2. Due to the fluoremeter restricted excitation light, $312 \mathrm{~nm}$ cannot generate a decent graph, therefore, excitation of $350 \mathrm{~nm}$ was used to obtain the spectra. 
Sample 1 to sample 5 has emission peaks at $430 \mathrm{~nm}, 471 \mathrm{~nm}, 511 \mathrm{~nm}, 548 \mathrm{~nm}$ and $511 \mathrm{~nm}$. The emission peak of sample 5 goes back to the same position as sample 3, this might be caused by the excitation light of $350 \mathrm{~nm}$ rather than $312 \mathrm{~nm}$.

Table 1: Summary of samples precursors and ethylene glycol amount.

\begin{tabular}{|l|l|l|l|l|l|}
\hline Sample & $\begin{array}{l}\text { Ethylene glycol (EG) } \\
(\mathrm{mmol})\end{array}$ & $\begin{array}{l}\text { Histidine } \\
(\mathrm{mmol})\end{array}$ & $\begin{array}{l}\text { Citric acid } \\
(\mathrm{mmol})\end{array}$ & $\begin{array}{l}\text { Ratio of carbon source to } \\
\text { EG }(\%)\end{array}$ & Particle size $(\mathrm{nm})$ \\
\hline 1 & 100 & 0.1 & 0.1 & 0.2 & $25 \pm 1.52$ \\
\hline 2 & 100 & 0.3 & 0.3 & 0.6 & $40 \pm 2.13$ \\
\hline 3 & 100 & 0.5 & 0.5 & 1. & $19 \pm 1.12$ \\
\hline 4 & 100 & 1 & 1 & 2 & $40 \pm 2.12$ \\
\hline 5 & 100 & 3 & 3 & 6 & $25 \pm 1.91$ \\
\hline
\end{tabular}

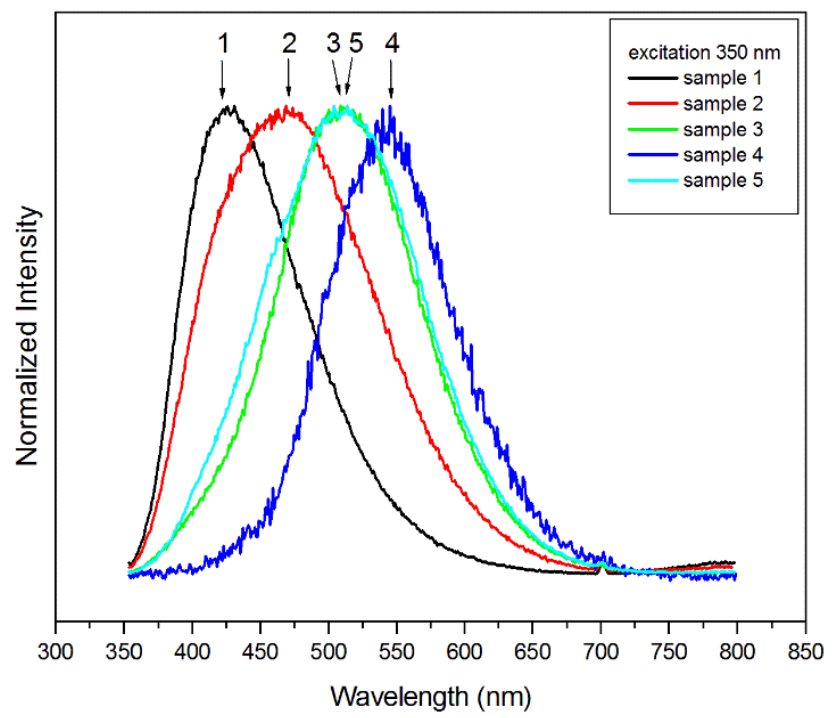

Fig. 2: Photoluminescence spectrum of color tunable carbon dots excited by $350 \mathrm{~nm}$.

The UV spectra of various carbon dots solution were measured and shown in figure 3. Typical absorbance curves of carbon dots were obtained similar to other research results. With increasing the ratio of Carbon source to EG, the emission of Cdots shows the read-shift.

TEM images of various carbon dots samples were measured, but there is not a clearly relationship between the carbon dots particle size and their fluorescence. Although there is reports of nanoparticles size causing fluorescence changes, obviously, this is not the situation. Though the mechanism for precursor amount induced fluorescence change is not yet fully understood. This facile optical tuneability provides an easy and environmental friendly method for producing certain colour range of carbon dots. Due the carbon composition of carbon dots, they normally possess high biocompatibility and very suitable for applications such as biosensing, bioimaging. Therefore, the following parts are focused on constructing biocompatible glucose biosensor based on sample 3 carbon dots. 


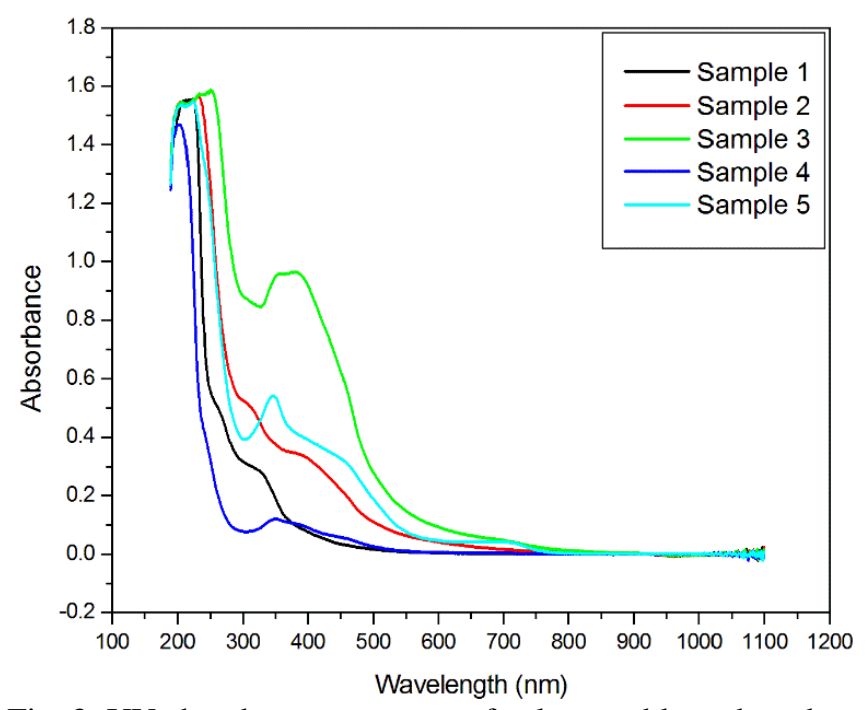

Fig. 3: UV absorbance spectrum of color tunable carbon dots.

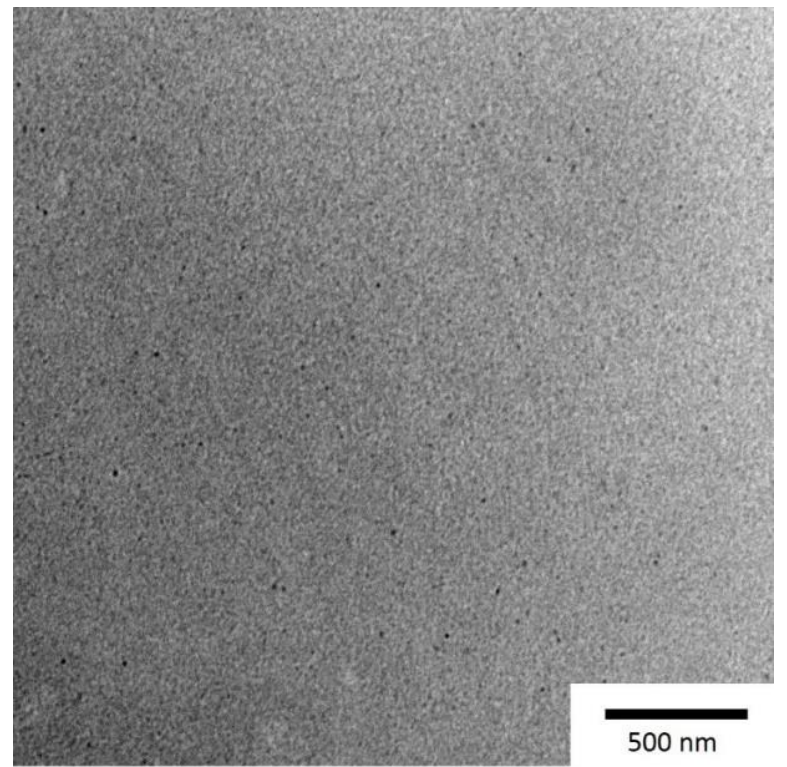

Fig. 4: TEM image of sample 3 carbon dots.

Figure 4 shows the TEM image of sample 3. It has an average particle size of about $19 \mathrm{~nm} \pm 1.2 \mathrm{~nm}$.

\section{Conclusion}

A facile microwave assisted polyol synthesis of carbon dots method has been developed. The average particle size is various in the range of 20-40 $\mathrm{nm}$ when the ratio of the precursor to EG increases. The photoluminescence of carbon dots shows tunable wavelength from $380 \mathrm{~nm}$ to $600 \mathrm{~nm}$ by adjusting the ratio of carbon precursors to ethylene glycol. The average particle size is estimated at Although the mechanism is not fully revealed, the tunable fluorescence of carbon-dots are related to the interfacial effect of the surface of carbon dots and passive surfactant.

\section{Acknowledgements}

Authors are thankful for the financial support from NSERC. 


\section{References}

[1] V. Georgakilas, J. A. Perman, J. Tucek, and R. Zboril, "Broad Family of Carbon Nanoallotropes: Classification, Chemistry, and Applications of Fullerenes, Carbon Dots, Nanotubes, Graphene, Nanodiamonds, and Combined Superstructures," Chem. Rev., vol. 115, no. 11, pp. 4744-4822, 2015.

[2] S. N. Baker and G. A. Baker, "Luminescent Carbon Nanodots: Emergent Nanolights," Angew. Chem. Int. Ed., vol. 49, no. 38, pp. 6726-6744, 2010.

[3] H. Li, Z. Kang, Y. Liu, and S.-T. Lee, "Carbon nanodots: synthesis, properties and applications," J. Mater. Chem., vol. 22, no. 46, pp. 24230-24253, 2012. DOI: 10.1039/C2JM34690G

[4] K. Hola, Y. Zhang, Y. Wang, E. P. Giannelis, R. Zboril, and A. L. Rogach, "Carbon dots-Emerging light emitters for bioimaging, cancer therapy and optoelectronics," Nano Today, vol. 9, no. 5, pp. 590-603, 2014.

[5] Y. Wang and A. Hu, "Carbon quantum dots: synthesis, properties and applications," J. Mater. Chem. C, vol. 2, no. 34, pp. 6921-6939, 2014. DOI: 10.1039/C4TC00988F

[6] Y. Fang et al., "Easy Synthesis and Imaging Applications of Cross-Linked Green Fluorescent Hollow Carbon Nanoparticles," ACS Nano, vol. 6, no. 1, pp. 400-409, 2012.

[7] J. Tang et al., "Carbon Nanodots Featuring Efficient FRET for Real-Time Monitoring of Drug Delivery and TwoPhoton Imaging," Adv. Mater., vol. 25, no. 45, pp. 6569-6574, 2013.

[8] C.-W. Lai, Y.-H. Hsiao, Y.-K. Peng, and P.-T. Chou, "Facile synthesis of highly emissive carbon dots from pyrolysis of glycerol; gram scale production of carbon dots $/ \mathrm{mSiO}_{2}$ for cell imaging and drug release," J. Mater. Chem., vol. 22, no. 29, pp. 14403-14409, 2012. DOI: 10.1039/C2JM32206D

[9] J. Zhang and S.-H. Yu, "Carbon dots: large-scale synthesis, sensing and bioimaging," Mater. Today, vol. 19, no. 7, pp. 382-393, 2016.

[10] P. Roy, P.-C. Chen, A. P. Periasamy, Y.-N. Chen, and H.-T. Chang, "Photoluminescent carbon nanodots: synthesis, physicochemical properties and analytical applications," Mater. Today, vol. 18, no. 8, pp. 447-458, 2015.

[11] S. Zhu, Y. Song, X. Zhao, J. Shao, J. Zhang, and B. Yang, "The photoluminescence mechanism in carbon dots (graphene quantum dots, carbon nanodots, and polymer dots): current state and future perspective," Nano Res., vol. 8, no. 2, pp. 355-381, 2015.

[12] A. M. Schwenke, S. Hoeppener, and U. S. Schubert, "Synthesis and Modification of Carbon Nanomaterials utilizing Microwave Heating," Adv. Mater., vol. 27, no. 28, pp. 4113-4141, 2015.

[13] X. Zhai et al., "Highly luminescent carbon nanodots by microwave-assisted pyrolysis," Chem. Commun., vol. 48, no. 64, pp. 7955-7957, 2012. DOI: 10.1039/C2CC33869F

[14] H. Zhu, X. Wang, Y. Li, Z. Wang, F. Yang, X. Yang, "Microwave synthesis of fluorescent carbon nanoparticles with electrochemiluminescence properties," Chem. Commun., no. 34, pp. 5118-5120, 2009. DOI: 10.1039/B907612C

[15] Y. Liu et al., "One-step microwave-assisted polyol synthesis of green luminescent carbon dots as optical nanoprobes," Carbon, vol. 68, pp. 258-264, 2014.

[16] X. Wang, K. Qu, B. Xu, J. Ren, and X. Qu, "Microwave assisted one-step green synthesis of cell-permeable multicolor photoluminescent carbon dots without surface passivation reagents," J. Mater. Chem., vol. 21, no. 8, pp. 2445-2450, 2011. DOI: 10.1039/C0JM02963G

[17] W. Wei, C. Xu, L. Wu, J. Wang, J. Ren, and X. Qu, "Non-Enzymatic-Browning-Reaction: A Versatile Route for Production of Nitrogen-Doped Carbon Dots with Tunable Multicolor Luminescent Display," Sci. Rep., vol. 4, p. 3564, 2014.

[18] H. Nie, et al., "Carbon Dots with Continuously Tunable Full-Color Emission and Their Application in Ratiometric pH Sensing," Chem. Mater., vol. 26, no. 10, pp. 3104-3112, 2014.

[19] H. Ding, S.-B. Yu, J.-S. Wei, and H.-M. Xiong, "Full-Color Light-Emitting Carbon Dots with a Surface-StateControlled Luminescence Mechanism," ACS Nano, vol. 10, no. 1, pp. 484-491, 2016.

[20] Y. Dong et al., "Carbon-Based Dots Co-doped with Nitrogen and Sulfur for High Quantum Yield and ExcitationIndependent Emission," Angew. Chem. Int. Ed., vol. 52, no. 30, pp. 7800-7804, 2013.

[21] S. Hu, A. Trinchi, P. Atkin, and I. Cole, "Tunable Photoluminescence Across the Entire Visible Spectrum from Carbon Dots Excited by White Light," Angew. Chem. Int. Ed., vol. 54, no. 10, pp. 2970-2974, 2015. 
[22] S. Zhu et al., "Highly Photoluminescent Carbon Dots for Multicolor Patterning, Sensors, and Bioimaging," Angew. Chem. Int. Ed., vol. 52, no. 14, pp. 3953-3957, 2013.

[23] L. Chen, W. H. Tse, A. Siemiarczuk, and J. Zhang, "Special properties of luminescent magnetic $\mathrm{NaGdF}_{4}: \mathrm{Yb}^{3+}, \mathrm{Er}^{3+}$ upconversion nanocubes with surface modifications," RSC Adv., 10.1039/C7RA03380J vol. 7, no. 43, pp. 2677026775, 2017.

[24] H. Dong, Y. C. Chen, and C. Feldmann, "Polyol synthesis of nanoparticles: status and options regarding metals, oxides, chalcogenides, and non-metal elements," Green Chem., 10.1039/C5GC00943J vol. 17, no. 8, pp. 4107-4132, 2015.

[25] L. Bao et al., "Electrochemical Tuning of Luminescent Carbon Nanodots: From Preparation to Luminescence Mechanism," Adv. Mater., vol. 23, no. 48, pp. 5801-5806, 2011.

[26] H. Dong, A. Kuzmanoski, Go, R. Popescu, D. Gerthsen, and C. Feldmann, "Polyol-mediated C-dot formation showing efficient $\mathrm{Tb}^{3+} / \mathrm{Eu}^{3+}$ emission," Chem. Commun., 10.1039/C4CC01715C vol. 50, no. 56, pp. 7503-7506, 2014.

[27] N. Gong et al., "Microwave-Assisted Polyol Synthesis of Gadolinium-Doped Green Luminescent Carbon Dots as a Bimodal Nanoprobe," Langmuir, vol. 30, no. 36, pp. 10933-10939, 2014/09/16 2014. 